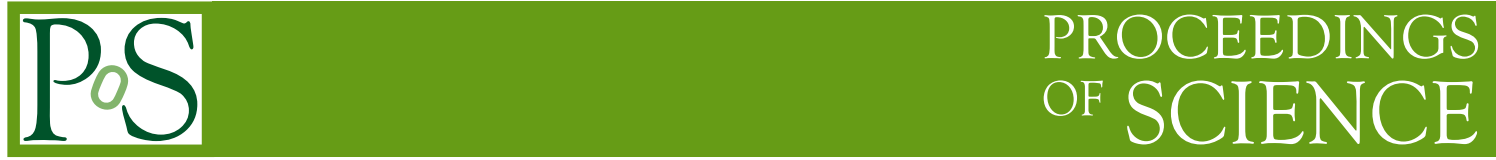

\title{
Dijet production in diffractive deep-inelastic scattering using proton spectrometers at HERA
}

\author{
Richard Polifka*† \\ Charles University in Prague \\ University of Toronto \\ E-mail: Richard.Polifka@cern.ch
}

The latest results from the $\mathrm{H} 1$ experiment on the diffractive dijet production in deep-inelastic scattering are presented. The first jet measurements with a tagged leading proton are described and the results are discussed in terms of diffractive parton densities and Regge factorisation.

36th International Conference on High Energy Physics,

July 4-11, 2012

Melbourne, Australia

\footnotetext{
${ }^{*}$ Speaker.

${ }^{\dagger}$ The work was supported by the grant SVV-2010-261 309.
} 


\section{Introduction}

An unique tool to investigate the nature of the object exchanged in diffractive interactions (the Pomeron) is provided through measurements of di-jets in diffractive deep inelastic scattering (DIS)[1]. Due to the presence of a hard scale - the transverse momenta of the jets - comparison of the measured data with next-to-leading order QCD calculations is allowed. This provides the possibility to test the QCD factorization in diffractive DIS as well as to look for effects of parton evolution beyond the DGLAP approach. In the following sections, the diffractive jet production with a proton measured with the $\mathrm{H} 1$ experiment at the HERA collider will be described and the latest results will be presented.

\section{Jets in Diffractive DIS at HERA}

In the high energy electron ${ }^{1}$-proton collisions at HERA, in the low $x_{B j}$ region, events of the type $e p \rightarrow e X Y$, where the final state consists of two systems $X$ and $Y$, comprise approximately $10 \%$ of all deep-inelastic scattering events (DIS). The products of the hard interaction with the photon emitted by the electron are contained in the system $X$, the system $Y$ contains the outgoing proton (elastic processes) or its low mass excitations (proton dissociation). Both systems are clearly separated by a region without energy flow (Large Rapidity Gap). These events are called diffractive. Diffractive interactions are described according to the Regge phenomenology in terms of the exchange of a colourless object which carries the quantum numbers of the vacuum, the so called Pomeron. Extensive measurements of inclusive diffractive DIS have been performed at HERA using two experimental methods of detecting diffraction - the Large Rapidity Gap ( $L R G)$ method [2] and the tagging of the outgoing proton with dedicated detectors (FPS and VFPS for H1, LPS for ZEUS) [1]. In addition to the standard DIS variables $Q^{2}$ (photon virtuality) and $x_{B j}$ (the longitudinal momentum fraction of the interacting parton with respect to the incoming proton), the following additional variables are used to describe diffractive processes: the fractional longitudinal momentum loss of the proton $x_{\mathbb{P}}=1-E_{p}^{\prime} / E_{p}$, the momentum fraction of the interacting parton with respect to the Pomeron $\beta$, defined similar to $x_{B j}$, and the squared four-momentum transfer at the proton vertex $t$. In the following measurements, the events tagged by the FPS and VFPS spectrometers are analysed.

The diffractive DIS cross section can be factorized (proven by Collins [3]) into a hard process (denoted as $d \hat{\sigma}^{e i}$ ) calculable within the pQCD framework and the diffractive parton distribution functions $f_{i}^{D}$ (DPDF) which have to be determined experimentally: $d \sigma\left(Q^{2},|t|, \beta, x_{\mathbb{P}}\right)=$ $\sum_{i} f_{i}^{D}\left(Q^{2},|t|, \beta, x_{\mathbb{P}}\right) \otimes d \hat{\sigma}^{e i}\left(Q^{2}, x_{B j}=x_{\mathbb{P}} \cdot \beta\right)$, where the sum runs over all partons. Regge factorization (also called proton vertex factorization) is usually assumed in addition, where the dependence on the variables characterizing the proton vertex $\left(x_{\mathbb{P}}\right.$ and $\left.t\right)$ factorizes from the hard interaction depending on $\beta$ and $Q^{2}: f_{i}^{D}\left(Q^{2},|t|, \beta, x_{\mathbb{P}}\right)=f_{\mathbb{I P} / p}\left(x_{\mathbb{P}}, t\right) \cdot f_{i}\left(\beta, Q^{2}\right)$, where the $f_{\mathbb{P} / p}$ stands for the Pomeron flux and $f_{i}$ is the probability of finding a parton $i$ in the Pomeron. The quark densities within the Pomeron are well constrained by the inclusive diffractive DIS measurements, whereas a better constraint on the gluon density in the Pomeron is provided by measurement of jets in the

\footnotetext{
${ }^{1}$ The term "electron" is used here to denote both electron and positron.
} 
final state. In addition, dijet measurements allow tests of perturbative QCD calculations, various models for modelling diffractive processes and serve as a tool for studying the parton evolution.

The measurements of jet production in diffraction with the FPS method [4] and VFPS method [5] have been performed for the first time. The cross section is corrected to the level of stable hadrons with a matrix $(F P S)$ and bin by bin (VFPS) unfolding. It is compared to pNLO DGLAP QCD calculations, DPDF-based RAPGAP Monte Carlo, two-gluon Pomeron RAPGAP Monte Carlo and soft colour interaction (SCI) model as implemented in the LEPTO Monte Carlo. The jet cross section is measured for two topologies. The 'two central jets' topology (see Table 1) is used for testing the proton vertex factorisation and the DPDFs in processes with a leading proton in the final state measure wirh both methods, whereas the 'one central + one forward jet' topology is motivated by the search for deviations from DGLAP parton evolution (FPS only). In contrary to non-diffractive DIS, jets reconstructed in the forward region are not spoiled by the presence of the proton remnant and can be therefore directly related to partons emitted during the interaction.

\begin{tabular}{|c|c|c|c|}
\hline Selection & VFPS two central jets & FPS two central jets & FPS one central + one forward jet \\
\hline DIS & $5<Q^{2}<80 \mathrm{GeV}^{2}$ & $4<Q^{2}<110 \mathrm{GeV}^{2}$ & $4<Q^{2}<110 \mathrm{GeV}^{2}$ \\
& $0.1<y<0.65$ & $0.05<y<0.7$ & $0.05<y<0.7$ \\
\hline Leading & $0.009<x_{\mathbb{P}}<0.024$ & $x_{\mathbb{P}}<0.1$ & $x_{\mathbb{P}}<0.1$ \\
Proton & $|t|<1 \mathrm{GeV}^{2}$ & $|t|<1 \mathrm{GeV}^{2}$ & $|t|<1 \mathrm{GeV}^{2}$ \\
\hline \multirow{4}{*}{ Jets } & $P_{T, 1}^{*}>5.5 \mathrm{GeV}$ & $P_{T, 1}^{*}>5 \mathrm{GeV}$ & $P_{T, c}^{*}, P_{T, f}^{*}>3.5 \mathrm{GeV}$ \\
& $P_{T, 2}^{*}>4 \mathrm{GeV}$ & $P_{T, 2}^{*}>4 \mathrm{GeV}$ & $M_{j j}>12 \mathrm{GeV}$ \\
& $-2.0<\eta_{1,2}<2.0$ & $-1<\eta_{1,2}<2.5$ & $-1<\eta_{c}<2.5$ \\
& \multicolumn{2}{|c}{} & $1<\eta_{f}<2.8, \eta_{f}>\eta_{c}$ \\
\hline
\end{tabular}

Table 1: Phase space of the diffractive dijet VBPS and FPS measurements, the asterisk marks variables reconstructed in the hadronic centre-of-mass system while other quantities are reconstructed in the laboratory frame.

The Figures 1 and 2 present the comparison of the measured hadron level cross sections to the NLO QCD DGLAP predictions for both methods for the 'two central jets' topology. The NLO predictions describe the data within quoted uncertainties. The Figure 3 presents the comparison of the measured hadron level cross sections to the Monte Carlo models; only the DPDF based RAPGAP MC is able to describe all shape aspects of the kinematic distributions. The Figure 4 left presents the comparison of the VFPS (data to NLO), FPS and LRG methods (direct comparison) and the extension of the phase space for the FPS method. All methods agree well within errors, the $L R G$ measurement is corrected for proton dissociation by a constant factor of 1.2. The measured $B$ slope of the $t$ distribution (right) is compared to the inclusive FPS measurement [1] (most right), the result is consistent with the assumption of the proton vertex factorisation being independent on the diffractive final state. Figure 5 presents the comparison of the data to NLO QCD DGLAP in the phase space enhancing effects beyond DGLAP. No deviations were observed within the uncertainties. 

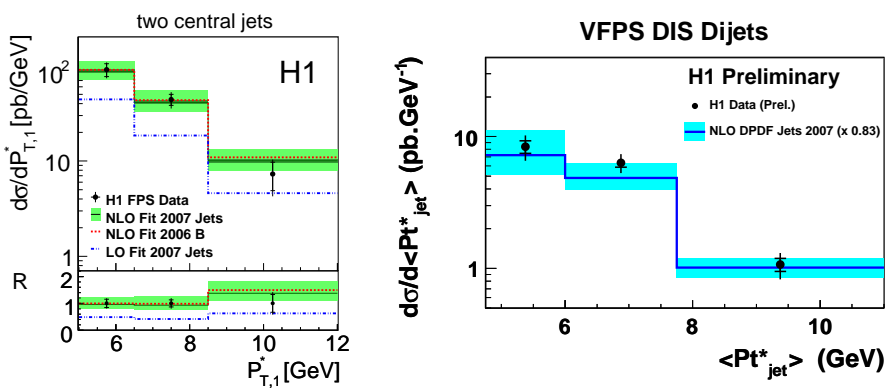

Figure 1: The sinlge differential cross section for the production of two central jets shown as a function of $p_{T, 1}^{*}$ for the FPS method (left) and $<p_{T, j e t}^{*}>$ for the VFPS method (right) compared to the NLO QCD DGLAP predictions based on DPDFs H1 2007 Jets and H1 2006 Fit B.
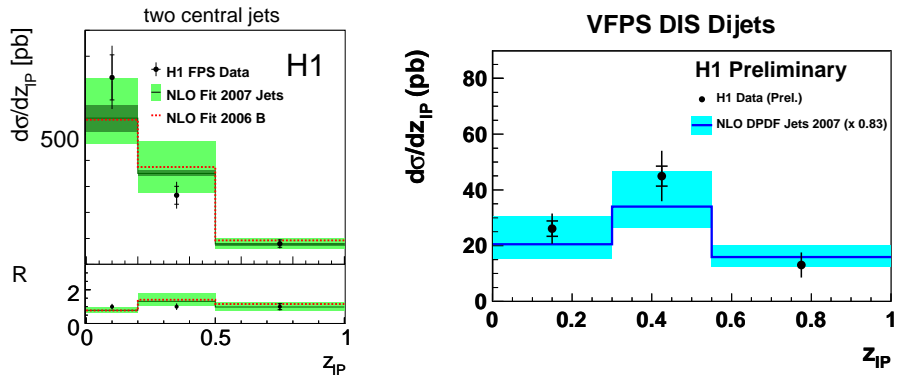

Figure 2: The sinlge differential cross section for the production of two central jets shown as a function of $z_{\mathbb{P}}$ for the FPS method (left) and VFPS method (right) compared to the NLO QCD DGLAP predictions based on DPDFs H1 2007 Jets and H1 2006 Fit B.

\section{Conclusion}

The latest results on the measurements of diffractive di-jet production with the scattered proton tagged in the Forward Proton Spectrometer or Very Forward Proton Spectrometer at HERA have been presented. The cross sections are well described by the NLO DGLAP QCD predictions even in the phase space enhancing possible deviations from the DGLAP parton evolution. A good agreement between the LRG and FPS methods as well as consistency with proton vertex factorisation assumption has been shown. The DPDF based RAPGAP Monte Carlo describes the kinematical distributions in shape, whereas the two-gluon pomeron and scoft colour interaction models fail.

\section{References}

[1] A. Aktas et al. [H1 Collaboration], Eur. Phys. J. C 71 (2011) 1578 [arXiv:1010.1476].

[2] C. Adloff et al. [H1 Collaboration], Z. Phys. C 76 (1997) 613 [hep-ex/9708016].

[3] J. Collins,Phys. Rev. D 57 (1998) 3051; [Erratum-ibid. D 61 (2000) 019902]; [hep-ph/9709499].

[4] F. D. Aaron et al. [H1 Collaboration], Eur. Phys. J. C 72 (2012) 1970 [arXiv:1111.0584].

[5] H1prelim-11-013, available from http://www-h1.desy.de/h1/publications/htmlsplit/H1 prelim-11-013.long.html 

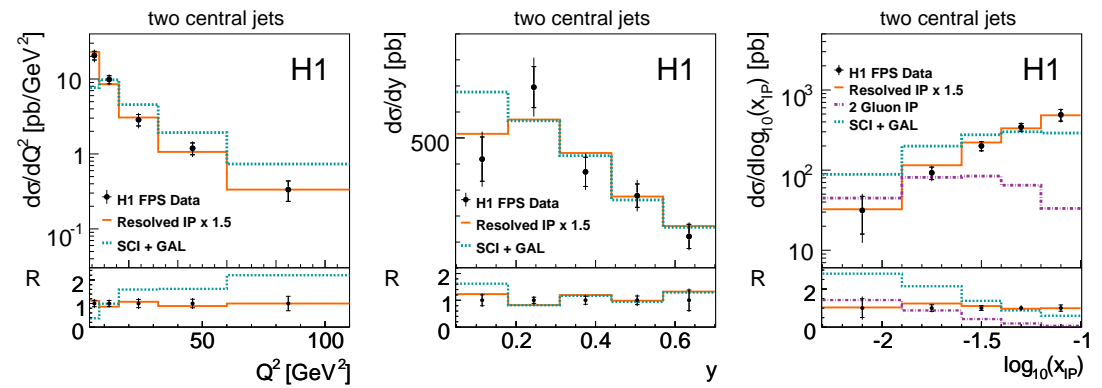

Figure 3: The sinlge differential cross section for the production of two central jets shown as a function of $Q^{2}, y$ and $\log \left(x_{\mathbb{P}}\right)$ compared to the DPDF based RAPGAP, 2 gluon $\mathbb{P}$ RAPGAP and SCI LEPTO Monte Carlo Models.
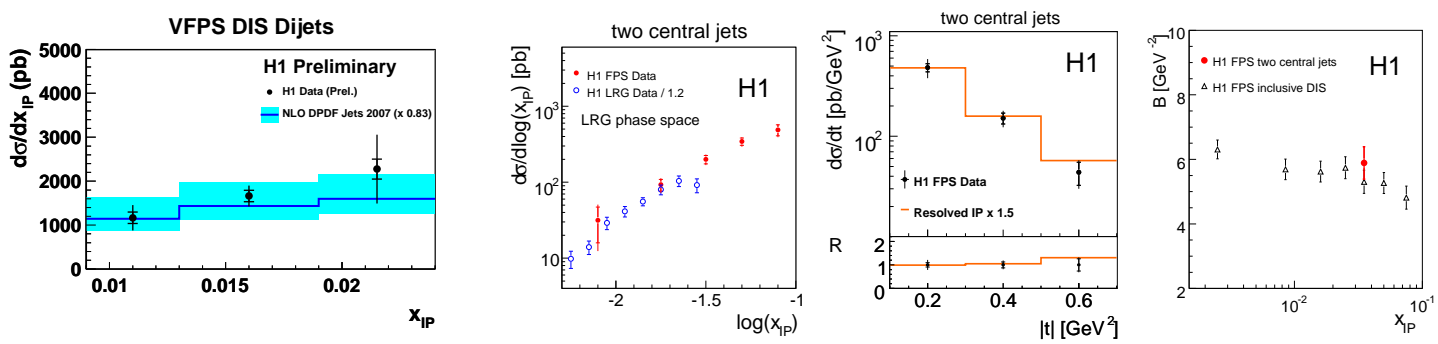

Figure 4: The single differential cross section measured in $x_{\mathbb{P}}$ with the VFPS method (most left) comparison of the FPS and LRG method as a fuction of $\log \left(x_{\mathbb{P}}\right)$ (left), the single differential cross section for the production of two central jets shown as a function of $t$ (right) and comparison of the fitted $B$ slope parameter in the inclusive (black open) and dijet (full red) diffractive measurements with tagged leading proton (most right).
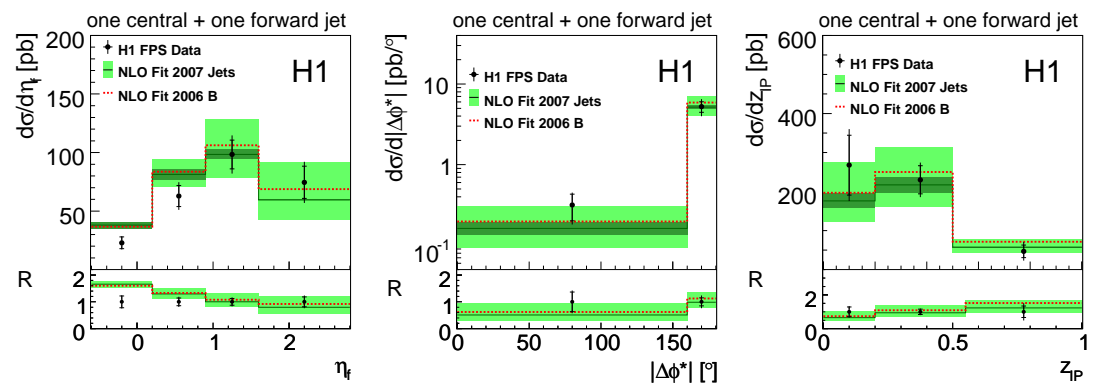

Figure 5: The sinlge differential cross section for the production of one central + one forward jet shown as a function of $\eta_{f}^{*},\left|\Delta \phi^{*}\right|$ and $z_{\mathbb{P}}$ compared to the NLO QCD DGLAP predictions based on DPDFs H1 2007 Jets and H1 2006 Fit B. 